\title{
Transverse Oscillations in Plasma Wakefield Experiments at FACET
}

E. Adli ${ }^{\mathrm{a}, \mathrm{b}}$, C. A. Lindstrøma ${ }^{\mathrm{a}, \mathrm{b}}$, J. Allen $^{\mathrm{b}}$, C. I. Clarke ${ }^{\mathrm{b}}$, J. Frederico ${ }^{\mathrm{b}}$, S. J. Gessner ${ }^{\mathrm{b}}$, S. Z. Green ${ }^{\mathrm{b}}$, M.J. Hogan ${ }^{\mathrm{b}}$, M. D. Litos ${ }^{\mathrm{b}}$, G. R.

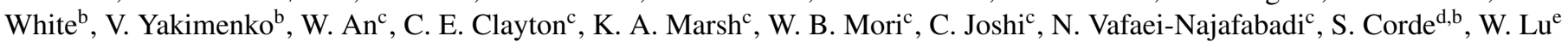

\author{
${ }^{a}$ Department of Physics, University of Oslo, N-0316 Oslo, Norway \\ ${ }^{b}$ SLAC National Accelerator Laboratory, 2575 Sand Hill Road, Menlo Park, California 94025, USA \\ ${ }^{c}$ University of California Los Angeles, Los Angeles, CA 90095, USA \\ ${ }^{d}$ LOA, ENSTA ParisTech, CNRS, Ecole Polytechnique, Université Paris-Saclay, 91762 Palaiseau, France \\ ${ }^{e}$ Department of Engineering Physics, Tsinghua University, Beijing 100084, China
}

\begin{abstract}
We study transverse effects in a plasma wakefield accelerator. Experimental data from FACET with asymmetry in the beam-plasma system is presented. Energy dependent centroid oscillations are observed on the accelerated part of the charge. The experimental results are compared to PIC simulations and theoretical estimates.
\end{abstract}

Keywords: electron beams, plasma wakefield, transverse dynamics, FACET User Facility

Wednesday $17^{\text {th }}$ February, 2016, 10:23

\section{Introduction}

In a plasma wakefield accelerator, a witness bunch is accelerated by the plasma wake driven by a driver bunch $[1,2]$. Preserving key beam parameters like emittance and energy spread during the acceleration process, is of fundamental importance for future accelerators based on plasma wakefield technology. In practical accelerators, some asymmetry in transverse beam profiles, as well as transverse jitter between driver and witness beams, will usually be present. Asymmetry in the driverwitness system may seed instabilities, including the hosing instability [3, 4, 5], and subsequently lead to emittance growth or beam break-up. In this paper we analyze experimental data from FACET [6] where the witness bunch is assumed to be gen- 28 erated by ionization injection [7]. In particular, we study the ${ }_{29}$ transverse phase space in data sets where we intentionally seed ${ }_{30}$ asymmetries at the injection. We compare the experimental re- ${ }_{31}$ sults to PIC simulations and theory.

\section{Experimental setup}

In the experiment a short $\left(\sigma_{z} \sim 20-40 \mu \mathrm{m}\right)$, high energy (20.3 GeV), high charge $(3 \mathrm{nC})$ electron driver bunch was sent ${ }^{37}$ into lithium vapour [8] with a vapour density of $8 \times 10^{16} \mathrm{~cm}^{-3}, 38$ 3 and a vapour length of $1.3 \mathrm{~m}$ FWHM. The transverse dimen- ${ }^{39}$ 24 sions of the bunch at the plasma interaction point (IP) were es- ${ }^{40}$ timated to some $10 \mathrm{~s}$ of $\mu \mathrm{m}$. The resulting space charge fields ${ }^{41}$ were strong enough to ionize the Li vapour [7]. No direct mea- ${ }^{42}$ surement was made of the actual length of the plasma channel ${ }^{43}$

Email address: Erik.Adli@fys.uio.no (E. Adli)

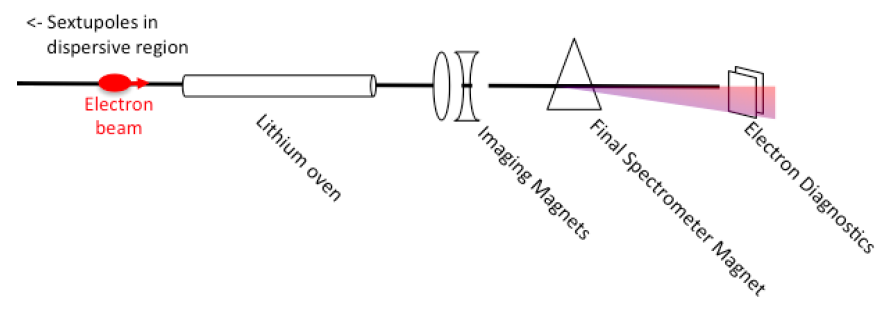

Figure 1: In the experiment a high charge, highly compressed drive bunch is sent into a lithium vapour. Electron charge may be trapped in the strong wake generated by the drive bunch, and accelerated to high energies. Sextupoles upstream of the plasma are used to generate dispersion at the plasma entrance, which may lead to transverse tilting of the drive bunch.

formed by the beam ionization. Numerical simulations, discussed in more details below, indicate that the drive bunch will have lost most of its energy after propagating through about 1 $\mathrm{m}$ of vapour. The ionized plasma electrons are blown out by the space charge fields of the driver, forming a plasma cavity. In [7] it is further shown that the electric fields may lead to ionization injection of electrons from the He buffer gas (used to contain the Li vapor), leading to trapped charge accelerated by the plasma cavity up to an energy of $\sim 30 \mathrm{GeV}$.

For the data presented in this paper, sextupoles on movers in dispersive regions upstream of the plasma IP were used to vary the horizontal dispersion at the entrance to the plasma. If the drive bunch is not fully compressed, the dispersion may induce a small drive bunch transverse tilt, and lead to asymmetries in the plasma cavity formation. The ionization injection may therefore take place at an offset with respect to the minimum of the transverse potential in the plasma cavity. Offset witness bunch injection may lead to deterioration of the transverse phase space. Figure 1 illustrates the experimental setup. 
We present data recorded by the FACET imaging spectrometer. The spectrometer consists of a dipole dispersing the beam according to energy, plus two quadrupoles which images (focuses) particles of selectable energy. The spectrometer images analyzed in this paper were recorded by a Cherenkov profile monitor [9]. In the data the accelerated charge is transversally straight on some shots, and show strong transverse oscillations on other shots. Figure 2 a) and b) show spectrometer images of shots without significant oscillations. Figure $2 \mathrm{c}$ ) and d) show spectrometer images of shots with strong oscillations along the energy axis. In the experimental shots presented the large amount of charge around the nominal energy of 20.3 $\mathrm{GeV}$ is believed to be drive bunch charge largely unaffected by the plasma. For the shots in Figure 2 the amount of observed charge in the accelerated part of the spectrum is a few 10s of pC. In the plasma accelerator blow-out regime [11], for low current witness bunches (weakly loaded wakes), the accelerating fields increase monotonically towards the end of the plasma cavity, which leads to a correlation between the energy and the position along an accelerated bunch; higher energy corresponds to further back along the bunch. According to PIC simulations with bunch and plasma parameters as assumed in the experiment, this relation holds, as shown in Figure $3 \mathrm{~b}$ ).

Data was taken for three different sextupole configurations, corresponding to a relative difference in the IP dispersion of + /$4 \mathrm{~mm}$. For each data set, the shots were sorted by hand according to whether there were clearly visible oscillations along the energy axis, or not. Figure 2 e) summarizes the number of shots with oscillations as function of sextupole position. In the data there is significant shot to shot variation within each data set, including shot to shot variation of the transverse profile of the bunch. However, there is still some degree of correlation between the percentage of oscillating shots and the relative dispersion at the plasma entrance.

In Figure $2 \mathrm{c}$ ) and d) the apparent amplitude of the transverse oscillations increases towards higher energy, assumed here to be towards the end of the bunch. A real amplification of the amplitude along the bunch would be consistent with an instability. A more careful analysis of the data reveals, however, that the observed amplification is consistent with aberration from the spectrometer imaging quadrupoles. Only one energy in each plane is imaged. For the imaged energy, the transport matrix element between a kick at the plasma exit, and a position at the spectrometer location, $m_{12}$, is by definition zero. For the data presented, the energy imaged is the nominal beam energy of $20.3 \mathrm{GeV}$. The black lines in Figure $2 \mathrm{c}$ ) and d) show the calculated aberrations, given by non-zero $m_{12}(E)$. When the aberrations are taken into account, the data shows no strong amplification of the oscillations along the bunch. Thus for the data presented here we observe no clear indication of transverse instabilities, despite the asymmetric injection process. a)
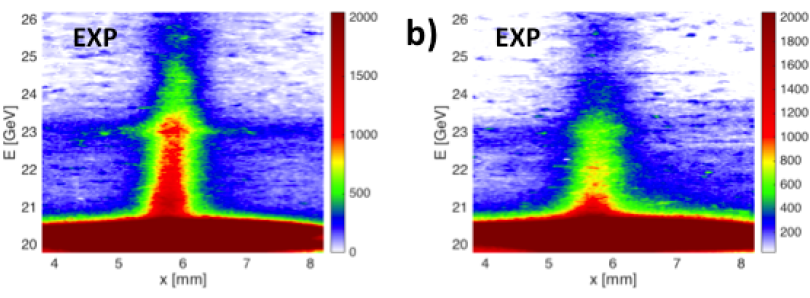

c)
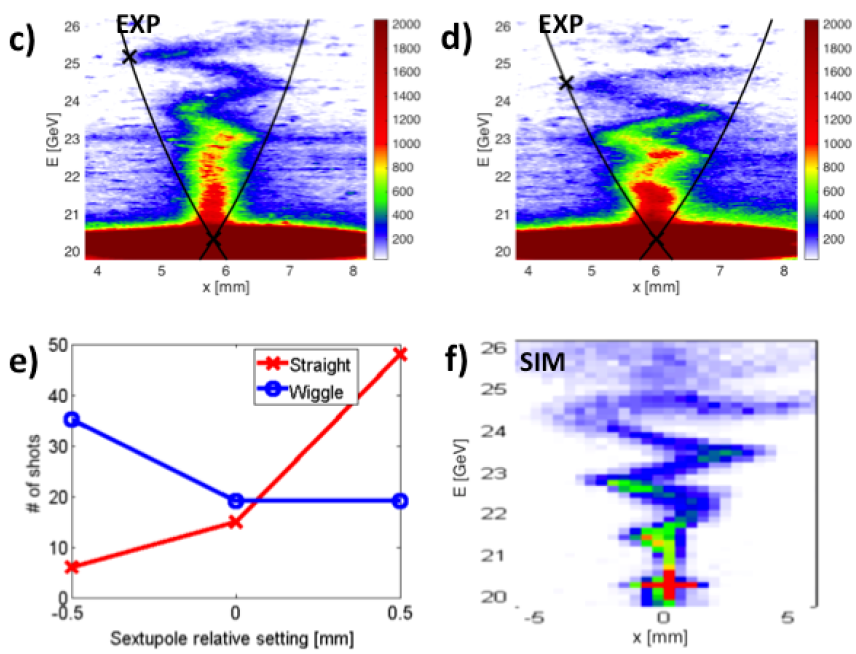

Figure 2: a) and b) show example spectrometer images of experimental shots where no significant transverse oscillations were observed. c) and d) show example spectrometer images of experimental shots where significant transverse oscillations were observed. The black lines indicate the aberration of the image due to the imaging quadrupoles. e) shows the correlation between the number of shots with oscillations and the setting of the upstream sextupoles. The sextupoles alter the dispersion at the plasma entrance. f) shows a simulated ionization-injected bunch after being tracked through a spectrometer line with the same quadrupole settings as in the experiment. 


\section{PIC simulations}

In order to further analyze the physics of the observed oscillations, we perform QuickPIC [12,13] simulations with beam and plasma parameters similar to the experimental parameters. The $20 \mathrm{GeV}$ drive bunch has $3 \mathrm{nC}$ of charge, $32 \mu \mathrm{m}$ initial spot sizes and a peak current of $12.8 \mathrm{kA}$. The beta functions at the plasma entrance are $\beta_{x}=0.5 \mathrm{~m}, \beta_{y}=5 \mathrm{~m}$ and $\alpha_{x}=\alpha_{y}$ $=0$. The bunch drives a wake where the electrons are fully blown out, as shown in Figure 3 a). A witness bunch is initially placed into the simulation, towards the back of the bubble where the accelerating fields are strong, in order to emulate a beam of trapped charge. The ionization process itself is not simulated. The witness bunch has $160 \mathrm{pC}$ of charge, $5 \mu \mathrm{m}$ spot sizes, a peak current of $3.8 \mathrm{kA}$, initial emittances of $5 \mu \mathrm{m}$ in both planes and an initial energy of $0.5 \mathrm{GeV}$. In the simulation both the drive bunch and witness bunch have Gaussian (symmetric) charge distributions in all three planes. The asymmetry in the system is emulated by transversally offsetting the witness bunch by a distance $\Delta x / \sigma_{x}=1 / 4$, where $\sigma_{x}=32 \mu \mathrm{m}$ is the rms spot size of the drive bunch. The vapour (neutral species) density is $8 \times 10^{16} \mathrm{~cm}^{-3}$, and the length of the vapor source is $1.3 \mathrm{~m}$. The simulation box has a size of $601 \mu \mathrm{m} \times 601 \mu \mathrm{m} \times 299 \mu \mathrm{m}$ in the two transverse dimensions and the longitudinal dimension, respectively. The number of the cells for the simulation box is $512 \times 512 \times 256$. As neither the details of the injection process, nor the full $6 \mathrm{D}$ phase space of the injected witness bunch are well known, some of the parameters have been tuned to match the simulation results to the experiment. In particular the bunch length of the witness bunch (a shorter witness bunch would have less energy spread) and the emittance of the witness 155 bunch (a larger emittance witness bunch would not be cleanly ${ }_{156}$ accelerated to $26 \mathrm{GeV}$ ) were tuned.

Figure 4 shows the evolution of the drive and witness bunch as they travel through the plasma. After $84 \mathrm{~cm}$ of plasma, the drive bunch has lost most of its energy in the plasma, while ${ }_{158}$ the witness bunch has gained more than $20 \mathrm{GeV}$. We observe ${ }_{159}$ that strong oscillations develop along the witness bunch as $i_{160}$ is accelerated. To further investigate the effect of spectrome- ${ }_{161}$ ter aberrations on the oscillations, the simulated witness bunch ${ }_{162}$ was tracked through a model of the spectrometer line, using the same quadrupole settings as in the experiment. The $\mathrm{x}-\mathrm{p}$ space ${ }^{163}$ for the simulated bunch (after $84 \mathrm{~cm}$ of plasma), at the spectrometer location, is shown in Figure $2 \mathrm{f}$ ). The agreement be- ${ }^{164}$ tween the simulated witness bunch and the accelerated part of ${ }^{165}$ the measured charge is good.

The offset witness bunch will undergo transverse betatron os- ${ }^{16}$ cillations [10] in the radial focusing fields of the plasma. The witness bunch does not significantly alter the longitudinal field ${ }^{168}$ (i.e. it does not load the wake), as can be seen in Figure 3b), since its peak current is much smaller than that of the drive ${ }^{169}$ bunch. The different longitudinal slices of the witness bunch ${ }^{170}$ therefore experience stronger accelerating fields towards the end of the bunch. Since the radial focusing fields in the blowout plasma cavity do not depend on the longitudinal position ${ }_{172}$ within the bubble [11], the phase advance of the betatron oscil-173 lations decreases towards the end of the bunch. The origin of $f_{174}$
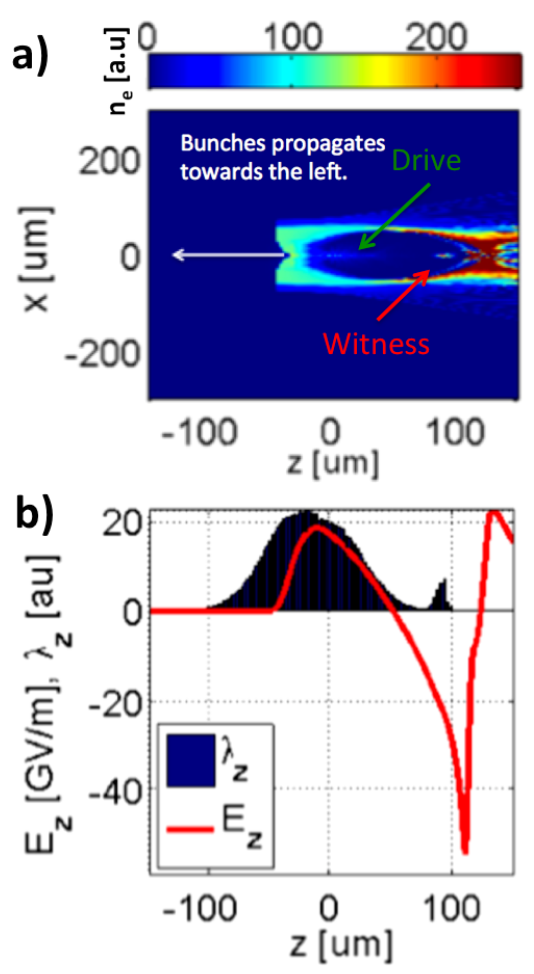

Figure 3: QuickPIC simulation. a) the electron density for the simulated drive bunch, the witness bunch and ionized plasma electrons. The bunches are traveling towards the left. $b$ ) The charge profile for the simulated bunches and the longitudinal wakefield along the center axis of the drive bunch.

the observed oscillations are therefore expected to be related to energy dependent phase advance along the bunch.

\section{Comparison to theory}

By assuming constant focusing fields in the plasma cavity, as expected in the blow-out regime, we can estimate the total phase advance for particles accelerated in the plasma, for a certain interaction length, as function of the energy gain, by solving Hill's equation [10],

$$
x^{\prime \prime}+K(s) x(s)=0 .
$$

Eq. 1 can be solved for each energy slice independently. The focusing term is given by the ion density, which in the blowout regime is equal to the plasma density $n_{0}$, and the particle Lorentz factor $\gamma$, as [11]

$$
K(s)=\frac{k_{p}^{2}}{2 \gamma(s)} .
$$

If the wake is static, the witness bunch energy will increase linearly, as

$$
\gamma(s)=\gamma_{0}+g_{\gamma} s,
$$

where $\gamma_{0}$ is the initial Lorentz factor and $g_{\gamma}$ is the accelerating gradient in units of Lorentz factors per meter. The simulation shows that the wake is close to static, suggesting that 


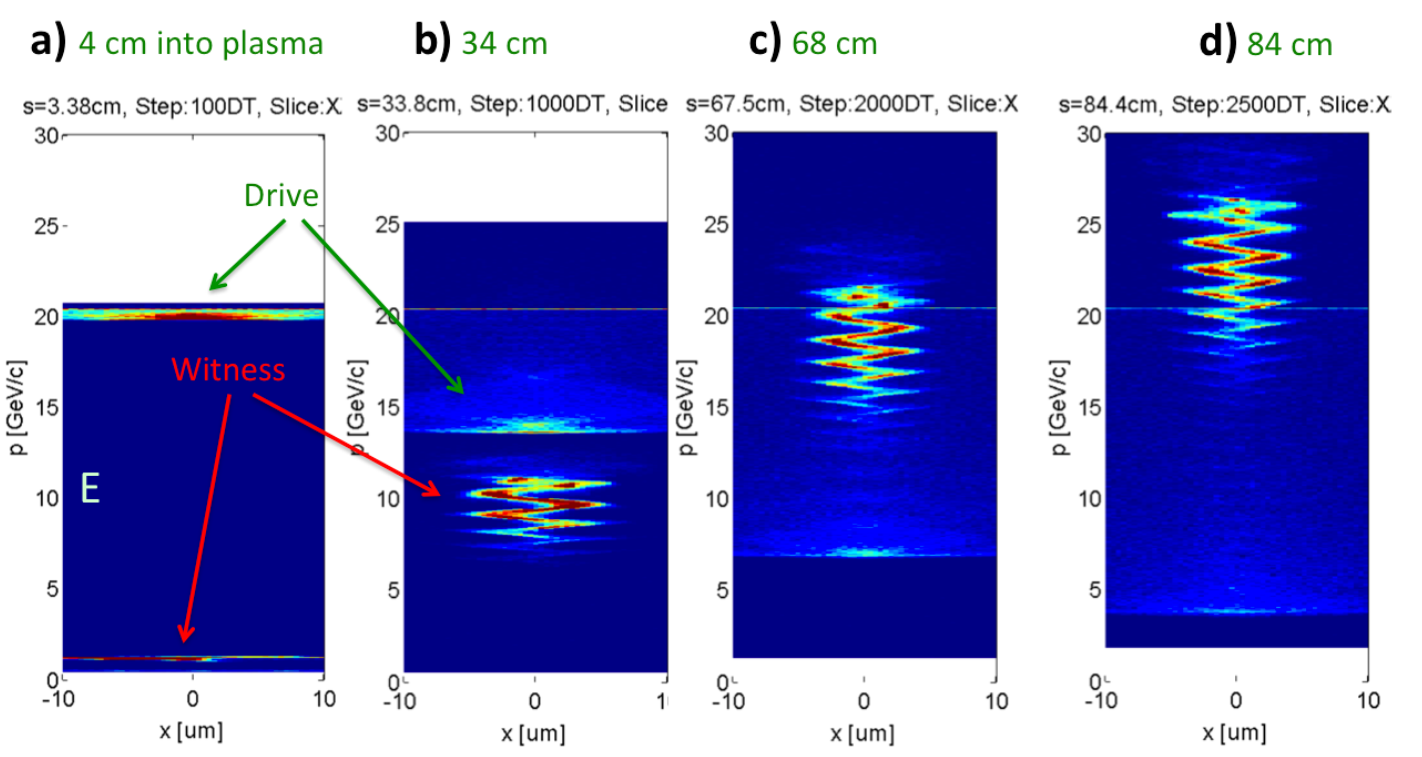

Figure 4: QuickPIC simulation. A witness bunch is injected with initial energy of $0.5 \mathrm{GeV}, 8 \mu \mathrm{m}$ offset with respect to the center of the bubble. This setup is meant to emulate a bunch injected off-axis, by ionization injection. The simulated $x$-p phase space, a) $4 \mathrm{~cm}$ into the plasma, b) $34 \mathrm{~cm}$ into the plasma, $c) 68 \mathrm{~cm}$ into the plasma, d) $84 \mathrm{~cm}$ into the plasma. As the witness bunch is accelerated, clear transverse oscillations are developing.

linear energy gain is a reasonable model. To estimate the number of centroid oscillations expected from energy dependent phase advance, we solve Eqs. (1-3) numerically, using that the bunch slices after $84 \mathrm{~cm}$ have a final energy from $20 \mathrm{GeV}$ to 26 $\mathrm{GeV}$, starting from an initial energy of $0.5 \mathrm{GeV}$. Apart from the change in relative focusing, the simple model used here does not take into account other effects of the acceleration. Figure 5 shows the final centroid position, as function of the energy gain, as estimated using Hill's equation. The result is that the centroid transverse position undergo five oscillations along the bunch slices accelerated from $0.5 \mathrm{GeV}$ to between $20 \mathrm{GeV}$ and $26 \mathrm{GeV}$. This is in excellent agreement with the number of oscillations observed in the PIC simulation, 4d), which is not unexpected. Comparing further to the experimental shots, shown in Figure $2 \mathrm{c}$ ) and d), the good correspondence between measured oscillations and calculated oscillations indicates that the acceleration in the experiment has indeed taken place in the blow-out regime.

In experiments with long drive bunches, the tail of the drive bunch itself may be accelerated, and may develop transverse 205 oscillations as well. The expected number of oscillations due to206 energy spread, for a drive bunch tail, is found by solving Eqs. 1-207 3 for an initial energy of $20 \mathrm{GeV}$ and final energies between $20_{208}$ $\mathrm{GeV}$ and $26 \mathrm{GeV}$. The result is 1.5 transverse oscillation along 209 the bunch. Comparing the two estimates of the transverse os-210 cillation frequency with the experimental results therefore gives 211 additional support to the findings in [7] that the accelerated part 212 of the spectrum is trapped charged rather than a part of the drive ${ }_{213}$ bunch.

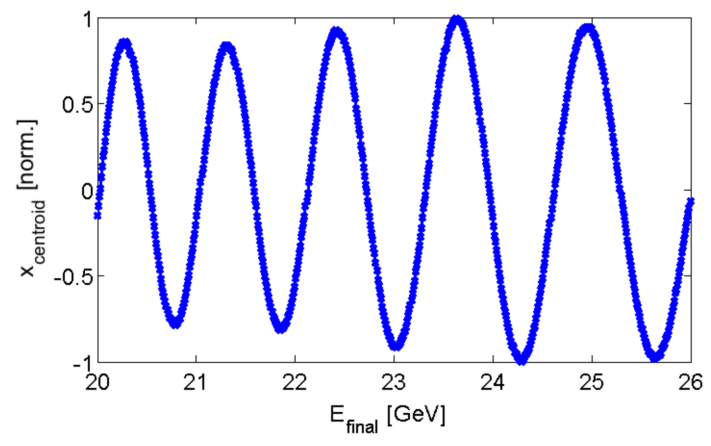

Figure 5: The centroid position of an offset witness bunch, after an interaction length of $84 \mathrm{~cm}$, as calculated by solving Hill's Equation for each energy slice. The calculation assumes constant absolute focusing forces and uniform acceleration in the plasma. This figure should be compared with Figure $4 d$ ).

We have analyzed the dynamics of injected charge in a plasma wakefield accelerator. Asymmetries in the driverwitness system were seeded by varying dispersion at the plasma entrance. Energy dependent oscillations were observed along the accelerated bunch. The oscillations can be largely explained by calculating the energy dependence of the centroid phase advance of the slice. Furthermore, we did not observe significant amplification of the oscillations with energy. Thus, for the data presented here no evidence for strong hosing or beam-breakup was found. 


\section{Acknowledgments}

Work at SLAC was supported by DOE contract number DE217 AC02-76SF00515 and also through the Research Council of ${ }_{218}$ Norway. Work at UCLA was supported by DOE grant num219 ber. DE-SC0010064 and the NSF grant number PHY-1415386. QuickPIC simulations used the Hoffman cluster at UCLA.

\section{$221 \quad$ References}

[1] P. Chen, J. Dawson, R. Huff and T. Katsouleas, Phys. Rev. Lett. 54, 693(1985)

224 [2] M. Litos et al. Nature 515, 92-95 (2014)

225 [3] D. H. Whittum et al., Phys. Rev. Lett. 67, 991 (1991)

226 [4] C. Huang et al., Phys.Rev.Lett. 99, 255001 (2007)

227 [5] E. Adli et al., in Proceedings of IPAC'12 (2012), p. 43

228 [6] M. J. Hogan et al., New J. Phys. 12, 055030 (2010)

229 [7] N. Vafaei-Najafabadi, submitted to PPCF, arXiv:1510.01402 (2015)

230 [8] P. Muggli et al., IEEE Trans. Plasma Sci. 27, 791-799 (1999)

231 [9] E. Adli, S.J. Gessner, S. Corde, M.J. Hogan, H.H. Bjerke, Nucl. Instrum. Methods Phys. Res. A 783, 35-42 (2015).

233 [10] E.D. Courant and H.S. Snyder, Annals Phys. 3 1-48 (1958)

234 [11] J.B. Rosenzweig, B. Breizman, T. Katsouleas, J.J. Su, Phys. Rev. A 44 $235 \quad$ R6189-R6192 (1991)

236 [12] C. Huang et al., J. Comput. Phys. 217658 (2006))

237 [13] W. An, V.K. Decyk, W.B. Mori, T. M. Antonsen Jr., J. Comput. Phys. 250 $238 \quad 165-177(2013)$. 